\title{
Influence of organic fillers on rheological behavior in phenol-formaldehyde adhesives
}

\author{
Xing Yang and Charles E. Frazier \\ Sustainable Biomaterials, Cheatham Hall, RM 230, Virginia Tech, 310 West Campus Dr., Blacksburg, \\ Virginia 24061, USA
}

\begin{abstract}
Phenol-formaldehyde resoles (PF) formulated with wheat flour and organic fillers have long been established for the manufacture of veneer-based wood-composites, and yet much remains unknown about these complex fluids. The rheology of PF/filler formulations was studied as a function of filler type and particle size. Corn cob (Zea mays) residue fillers behaved differently from those made from alder bark (Alnus rubra) and walnut shell (Juglans regia). It was shown that viscoelastic network structures formed within the liquid formulations as a function of shear history, filler type, and filler particle size. The precise nature and origin of these effects is unknown but could involve disintegration of filler particle aggregates on a non-colloidal scale, and/or colloidal effects within the liquid PF medium. In the latter case colloidal structures could form among associated PF chains and also from proteins, polysaccharides, and lignins that leach from wheat flour and filler particles. Relative to alder bark and walnut shell, the unique behavior of corn cob residue was discussed with respect to chemical composition. Many implications for impact on industrial practice are feasible and should be the subject of future research.
\end{abstract}

Keywords: Adhesives for wood (A); Phenolic (A); Rheology (C); Organic filler

* Corresponding author

Department of Sustainable Biomaterials

Virginia Tech, 230 Cheatham Hall

Blacksburg, VA 24061, USA

Phone: +1 (540) 231-8318

E-mail: cfrazier@vt.edu 


\section{Introduction}

Phenol formaldehyde (PF) resoles are outstanding wood adhesives that remain as the preferred resin used to manufacture structural wood-based composites from veneer, as in plywood and laminated veneer lumber. In such veneer applications PF resoles are commonly formulated with fillers and extenders [1]. Definition of the terms "filler" and "extender" vary [2], but in the wood products industry fillers are considered to be generally inert, while extenders are proteinaceous and amylaceous materials that exhibit some intrinsic adhesive properties [3]. Contemporary PF/filler/extender formulations are designed to meet a variety of performance criteria including bulk flow, prepress tack, precure moisture retention, gap-filling properties, not to mention postcure strength and durability [1, 4].

For the applications described here, the fillers are typically organic flours derived from lignocellulosic biomass waste streams such as walnut nutshell (Juglans regia), red alder (Alnus rubra) bark, and furfural production residues, i.e. corn cob residue [1]. While these fillers have held commercial significance for several decades, they have been the subject of little or no detailed analysis resulting in scientific publication. Only Ebewele et al. [5] reported the impact that walnut shell fillers had on adhesive performance. This publication represents an industry/university cooperative research effort intended to broaden the scientific base underlying current and future technologies. The organic fillers studied here, flours of walnut shell, alder bark, and corn cob (furfural production) residue impact the flow properties of PF adhesives. Accordingly, this is a report of how liquid PF adhesive rheology is impacted by the type and particle size of organic filler.

\section{Experimental}

\subsection{Materials}

Modal $^{\mathrm{TM}}$ alder bark (A) filler, walnut shell (W) filler, corn cob residue (C) filler, and wheat flour extender were kindly provided by Willamette Valley Company (Eugene, OR, USA). The walnut tree species was Juglans regia, English walnut, representing an unknown mixture of tree varieties characteristic of commercial production in northern California, U.S.A. The subspecies of corn (Zea mays) was unknown. Phenol formaldehyde (PF) resin was a Cascophen ${ }^{\mathrm{TM}}$ plywood resin, supplied by Momentive Specialty Chemicals $(\mathrm{pH}=11 \sim 12$, solids content $=43 \%$ ). Sodium carbonate (powder) and $50 \%$ sodium hydroxide (liquid), used for adhesive formulation, were 
obtained from Willamette Valley Company (Eugene, OR, USA) and Fisher Scientific, respectively.

\subsection{Filler size classification}

The fillers were classified into sub-samples by sieves. The classified fillers samples $(A, W$, or $C)$ were numerically coded as 1, 2, or 3: 1, small size range (passing through 325 mesh); 2, medium (passing through 200 mesh but retained on 325 mesh); 3, large size range (passing through 100 mesh but retained on 200 mesh). Size distributions of the classified fillers, dispersed in isopropanol, were analyzed using a Partica LA-950 laser diffraction particle size distribution analyzer (HORIBA Scientific).

\subsection{Chemical compositional analysis}

Large size (3) fillers were used for chemical compositional analyses including the determination of extractives, alkaline leachates, acid insoluble (Klason) and acid soluble lignin, and sugars. The extractive content was determined according to ASTM D1105 [6]. The extractive-free sample was denoted as Ex-free filler. Approximately $5 \mathrm{~g}$ dry Ex-free filler was extracted with 125 $\mathrm{mL}$ sodium hydroxide solution ( $4 \mathrm{wt} \%$ ) for 24 hours. The solid fraction of the mixture was collected by centrifugation and washed with deionized water until neutral. The residue, denoted as Ex\&AE-free filler, was vacuum dried $\left(45^{\circ} \mathrm{C}, 5.4 \mathrm{mmHg}\right)$. Lignin and carbohydrate content in Ex-free and Ex\&AE-free samples were determined respectively according to NREL/TP-51042618 and NREL/LAP-004 [7, 8]. Lignin and sugar contents in filler alkaline leachates were estimated by taking the differences in the chemical compositions of Ex-free and Ex\&AE-free samples.

\subsection{Adhesive formulation}

Sub-samples were formulated with PF resins with the addition of $\mathrm{Na}_{2} \mathrm{CO}_{3}, 50 \% \mathrm{NaOH}$, water, and wheat flour as shown in Table 1 (Willamette Valley Company, Eugene, OR, USA). The moisture contents of fillers and wheat flour in the formulation, measured with an Ohaus moisture analyzer, were 5-8 \%, and $10 \%$, respectively. Each formulation (800 g) was prepared in a mixer (5 quart bowl-lift stand mixer, KitchenAid ${ }^{\circledR}$ ) with a speed of 75 rpm ("stir" speed level) at room temperature. The formulated adhesives were denoted as A-1, A-2, A-3, W-1, W-2, W-3, C-1, C2 , and C-3 respectively. Additionally, the influence of the wheat flour extender was observed by preparing a formulation as in Table 1, but that no fillers were included (PF-no filler). Within 
complete adhesive formulations, the wheat flour volume fraction was $3.6 \%$ and filler volume fractions were $4-6 \%$.

\section{Table 1}

PF adhesive formulation (and mixing procedure).

\begin{tabular}{lc}
\hline Formulation contents & weight \% \\
\hline Water & 18.4 \\
Filler & 7.5 \\
$\quad$ (Mix 2 min.) & 22.8 \\
$\begin{array}{l}\text { PF resin } \\
\quad \text { (Mix 2 min.) }\end{array}$ & 5.5 \\
$\begin{array}{l}\text { Extender (wheat flour) } \\
\quad \text { (Mix } 8 \text { min.) }\end{array}$ & 3.0 \\
$\begin{array}{l}\text { Sodium hydroxide, 50\% } \\
\quad \text { (Mix 10 min.) }\end{array}$ & 0.5 \\
$\begin{array}{l}\text { Sodium carbonate } \\
\quad \text { (Mix 1 min.) }\end{array}$ & 42.3 \\
$\begin{array}{l}\text { PF resin } \\
\quad \text { (Mix 2 min.) }\end{array}$ & 100 \\
Total mixture &
\end{tabular}

\subsection{Rheological analysis}

Rheological flow-curves were obtained for all adhesive formulations (as a function of filler type and particle size), and flow-curves were also obtained for the base resin (neat PF), and the complete adhesive formulation minus the added filler (PF-no filler). A concentric cylinder geometry (conical rotor: $14 \mathrm{~mm}$ radius, $42 \mathrm{~mm}$ height; cup: $15 \mathrm{~mm}$ radius; gap: $1 \mathrm{~mm} ; 25^{\circ} \mathrm{C}$ ) was employed on a TA instruments AR G2 rheometer. For all adhesive formulations, the flowcurves were obtained immediately after formulation mixing. The rheological analysis involved a two-step acquisition of sequential, steady-state flow curves as follows: Step 1) using no specimen pre-shear, steady-state flow analysis with increasing shear rate from 0.05 to $4000 \mathrm{~s}^{-1}$, and Step 2) steady-state flow analysis under decreasing shear rate from 4000 to $0.05 \mathrm{~s}^{-1}$; the transition between steps 1 and 2 was immediate with no intervening equilibration time. The steady-state criterion was defined as less than $5 \%$ change in shear stress among three consecutive data points over a period not longer than 1 minute. All data met the criterion except those at shear rates less than $0.08 \mathrm{~s}^{-1}$, which has been excluded.

Additional experiments were conducted on specimens A-1 and C-3 using the two-step procedure described above, but with additional segments $(X, Y$, and $Z)$ to create the following sequence: Segment $X$; Step 1 (ramp-up) flow curve; Segment Y; Step 2 (ramp-down) flow curve; 
Segment Z. The segments $X, Y$, and Z contained a creep/recovery (0.05 Pa, 60 s.; $0.0 \mathrm{~Pa}, 60$ s.), followed by a frequency sweep $(0.01-0.5 \mathrm{~Hz}$; 0.05 Pa). The 0.05 Pa stress applied in segments $X, Y$, and $Z$ was well within the linear response (as determined from an oscillatory, 1 $\mathrm{Hz}$, stress sweep).

\section{Results and discussion}

\subsection{Filler size distribution}

The size distributions of unclassified fillers and wheat flour are shown Fig. 1. Three fillers were classified into three size ranges using the same sieves for each filler-type. Fig. 2 demonstrates that the large and medium size fractions were very comparable among filler types. Whereas the small size fraction exhibited some variation among filler types, possibly indicating geometric differences that might arise from cellular anatomical differences among the three tissue types, alder bark, walnut shell, and corn cob.

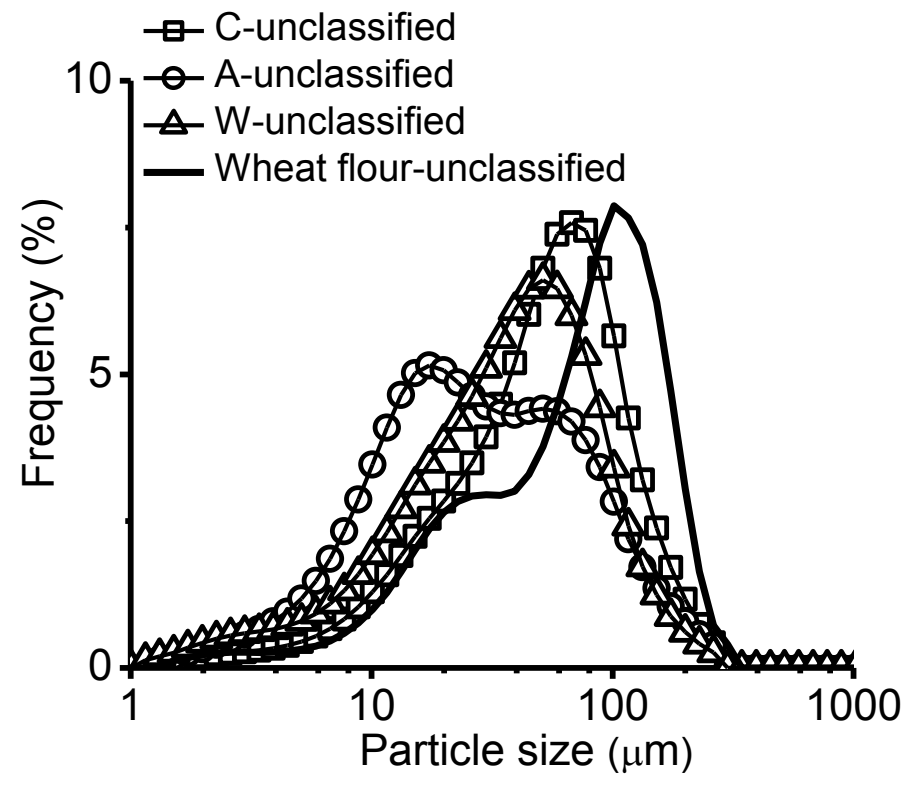

Fig. 1. Particle size distribution of unclassified fillers and wheat flour. 

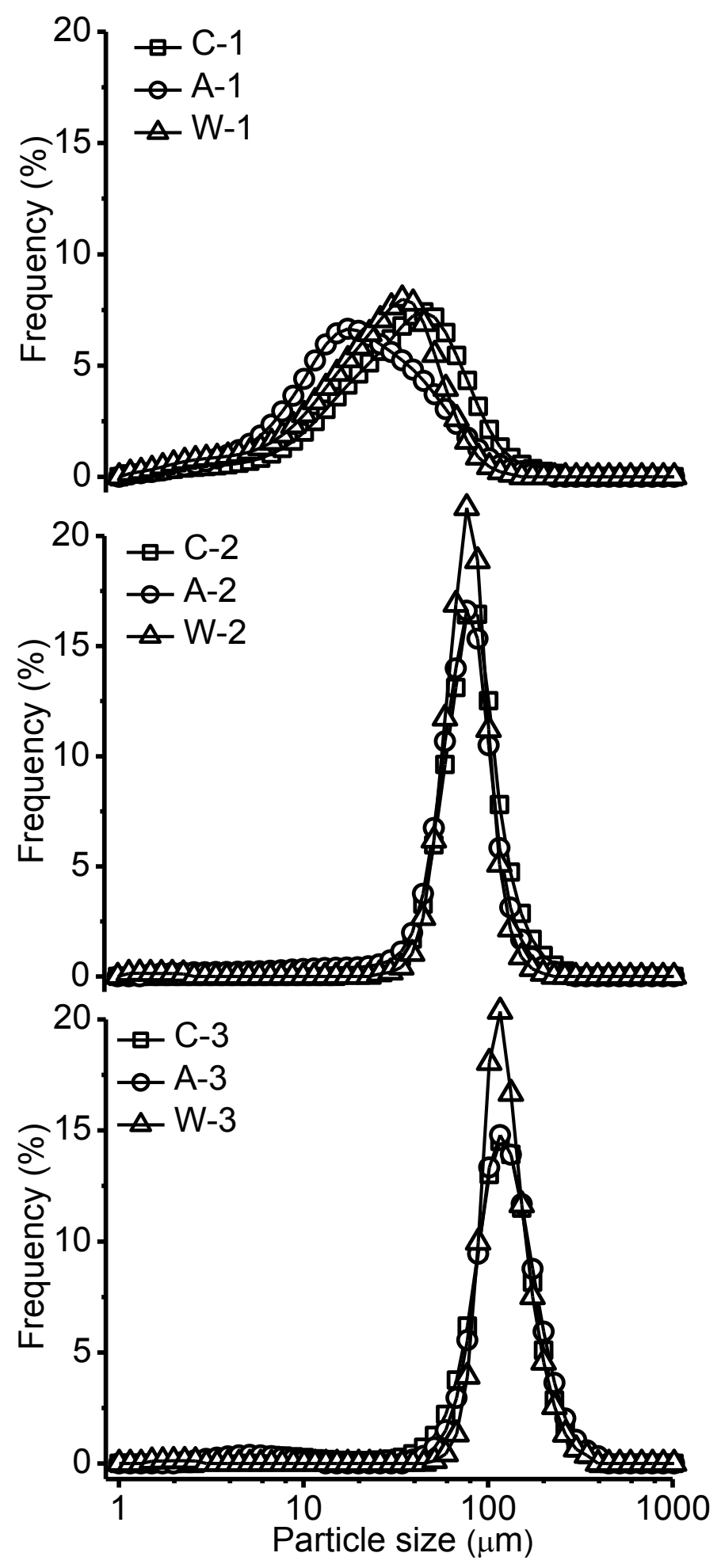

Fig. 2. Particle size distributions of classified fillers. 


\subsection{Rheology of neat PF resin and PF-no filler under increasing shear rate}

Flow curves (increasing shear rate) of the base resin (neat PF) and of the adhesive formulation without filler (PF-no filler) are shown in Fig. 3. The neat PF resin was principally Newtonian, but shear-thinning was observed at shear rates above $1250 \mathrm{~s}^{-1}$. According to its viscosity and polymer concentration, the neat PF resin probably exhibited chain entanglement [9]; however, this topic was not studied. Consequently, the shear-thinning could reflect chain disentanglement and/or the dissociation of phenolic interactions [1, 9]. In contrast, PF-no filler exhibited a Newtonian plateau but with more gradual shear thinning over a broader shear rate range. The wheat flour particles ( $3 \%$ volume fraction in this specimen) are known to release proteins and polysaccharides under the highly alkaline conditions in the PF resin [10-12].

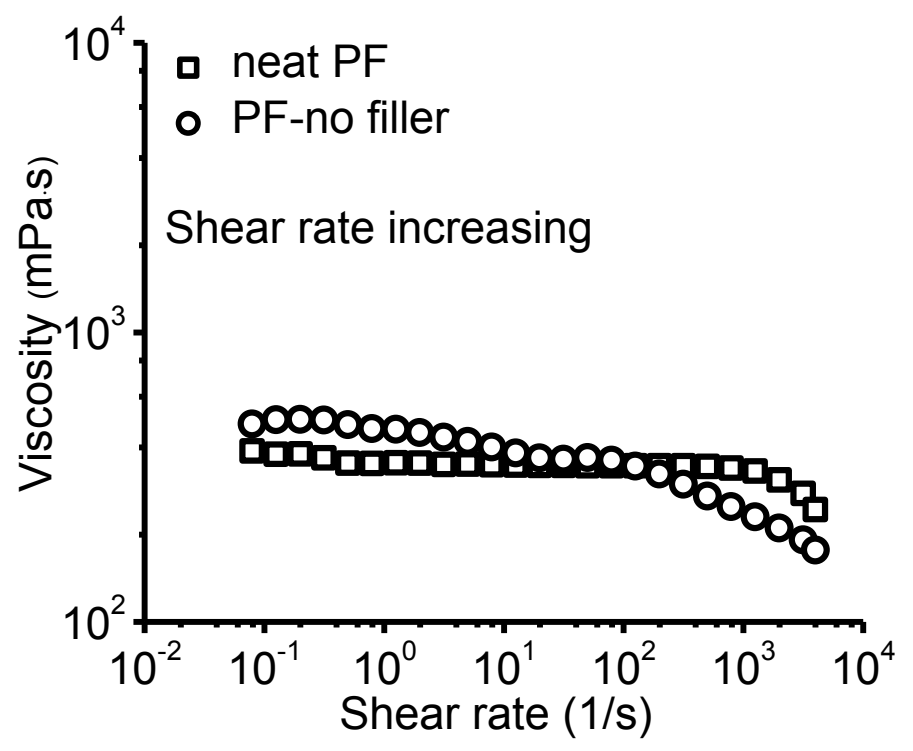

Fig. 3. Flow curves (increasing shear rate) of neat PF and PF-no filler.

\subsection{Rheology of PF with classified fillers}

Complete adhesive formulations (varying by filler type and particle size) were subjected to a two-step rheological analysis. Without pre-shear, analysis began by ramping-up the shear rate (Step 1), immediately followed by ramping-down the shear rate (Step 2) with no intervening equilibration or delay, Fig. 4. Comparison of Fig. 3 and 4 (shear rate increasing) reveals that all fillers produced more complex flow behavior as compared both to the base resin and the formulation without fillers (PF-no filler). Under increasing shear rate, all three filler types exhibited a generally similar behavior: shear thinning at low shear rates, followed by an intermediate plateau, and shear thinning at the highest shear rates. As the shear rate was ramped-down (Step 2), all formulations regained viscosity in a recovery that was principally 
monotonic (i.e. W-2), or that exhibited two distinct regions with remarkably rapid recovery at lower shear rates (i.e. A-1). Three of the corresponding hysteresis curves are shown in Fig. 5, which crudely indicate the presence or absence of structural reorganization [13, 14]. In Fig. 5 no significant reorganization is seen in C-3 and W-2; the hysteresis curves generally formed closed loops where the initial pre-shear structure appeared to reform (thixotropy is not implied, nor of concern here). In contrast, A-1 exhibited cross-over behavior in the low shear region which is suggestive of structural reorganization $[13,14]$. Among the particle size effects observed in Fig. 4 , the most dramatic were seen for the large corn cob residue particles (C-3) in Step 1 (ramping-up), and for the small alder bark and walnut shell particles (A-1, W-1) in Step 2 (ramping-down). All three specimens exhibited a similar Step 2 recovery process, but C-3 does not restructure whereas A-1 does (and perhaps also W-1; not determined). 

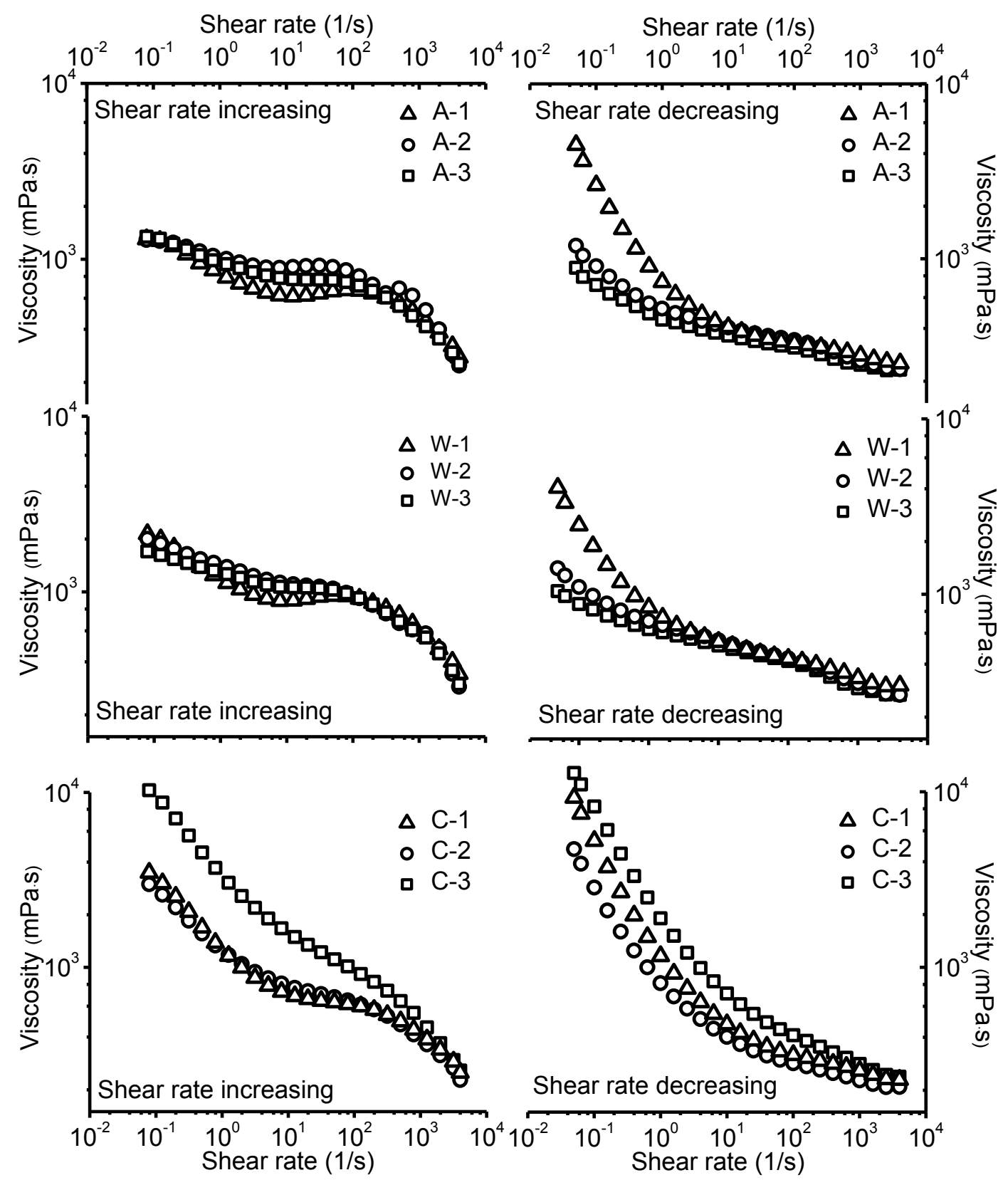

Fig. 4. Rheological behavior of PF with classified fillers under a two-step acquisition. 


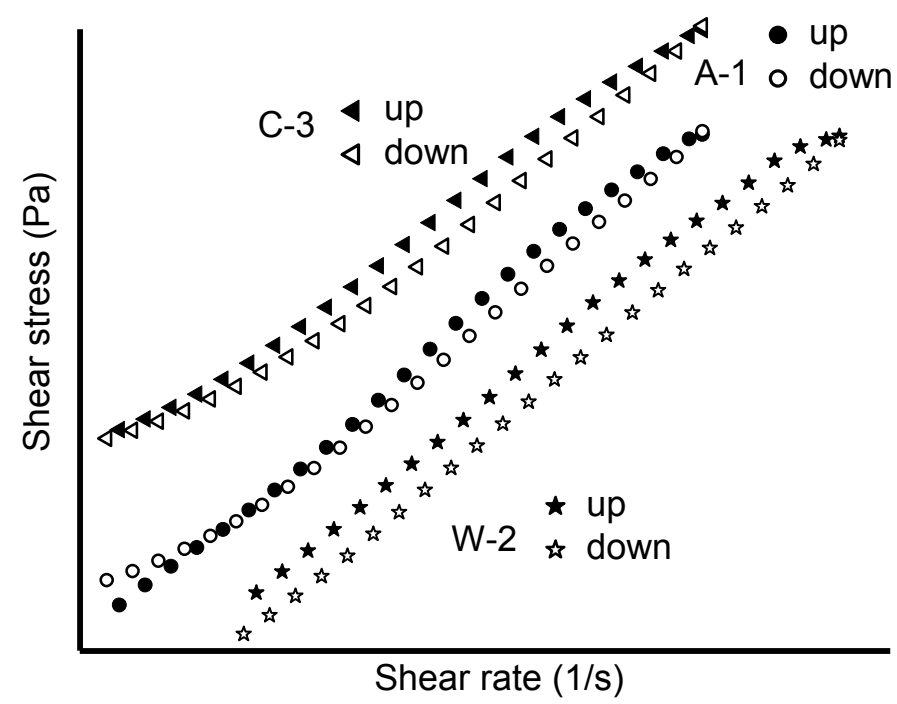

Fig. 5. Hysteresis curves for select PF/filler formulations, all scaled identically but shifted for clarity.

Formulations A-1 and C-3 were subjected to more detailed analysis. In this case the same twostep flow was imposed, however three additional segments were added (named $X, Y$, and Z). Segment $X$ occurred just prior to Step 1 (ramping-up); segments $Y$ and $Z$ respectively occurred immediately before and after Step 2 (ramping-down). Segments $X, Y$, and Z contained a 120 second creep/recovery followed by a frequency sweep; the applied stresses $(0.05 \mathrm{~Pa})$ were well within the linear response (determined separately using a $1 \mathrm{~Hz}$ stress sweep). Fig. 6 indicates that the pre-shear character of formulation A-1 was principally viscous (segment $X$ ); the creep response was nearly linear and very little creep recovery occurred. Subsequent shearing during Step 1 flow caused increased elasticity (segment $Y$ ), which developed further as the viscosity recovered during Step-2 decreasing shear flow (segment $Z$ ). The restructuring in formulation A-1 (seen in Fig. 5) apparently involved a transformation from mostly viscous to remarkably viscoelastic behavior, suggesting the development of some type of network structure. In contrast to A-1, the pre-shear nature of formulation C-3 was viscoelastic, and the two-step flow process caused a more dramatic development of elastic behavior (note scale difference in Fig. 6). Relative to $A-1$, note how the $C-3$ responses are juxtaposed with segment $X$ showing elasticity that is intermediate of segments $Y$ and $Z$. 

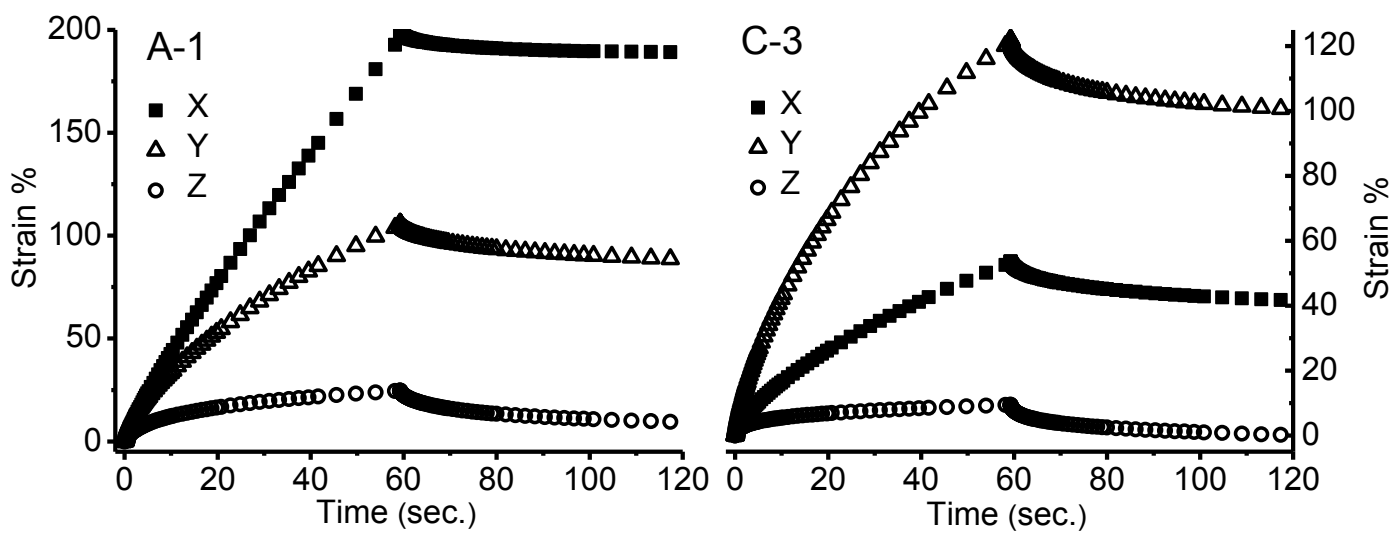

Fig. 6. Creep/recovery responses, respectively $X$ and $Y$, occurring before and after Step 1 ramping-up flow; $Z$ occurred after Step 2 ramping-down flow. Note the different scales for samples A-1 (left) and C-3 (right).

Fig. 7 shows the corresponding frequency sweeps occurring after the creep/recovery tests. As above, the pre-shear nature of $A-1$ was mostly viscous where in segment $X$ the loss modulus was greater than the storage modulus across the frequency range employed. Attending the transformation in A-1 was an increase in the storage and loss moduli; and the storage and loss were effectively equal over the lowest frequencies (segments $Y$ and $Z$ ). The very low moduli observed in A-1 (segment Z) are indicative of high fluidity, but the equivalence of the storage and loss moduli are also suggestive of network structure in this viscoelastic fluid. Regarding formulation C-3, careful inspection of Fig. 7 reveals that the frequency sweeps in segments $X$ and $Y$ were essentially identical; the loss moduli always remained above the storage moduli, but the loss and storage very nearly converged at about $0.1 \mathrm{~Hz}$ (in Fig. 6, the greater elasticity of C3 compared to A-1 is explained by comparing the ordinate scales in Fig. 7). However, segment Z shows a cross-over, also near $0.1 \mathrm{~Hz}$, where below that frequency the storage exceeds the loss. Note for formulation $\mathrm{C}-3$ that all moduli in segments $\mathrm{X}$ and $\mathrm{Y}$ fall off (trend towards increasing slope) over the low frequency range; whereas in segment $Z$ the moduli trend towards plateau behavior. The tendency towards a low frequency plateau indicates greater network development in C-3 as compared to formulation A-1. 


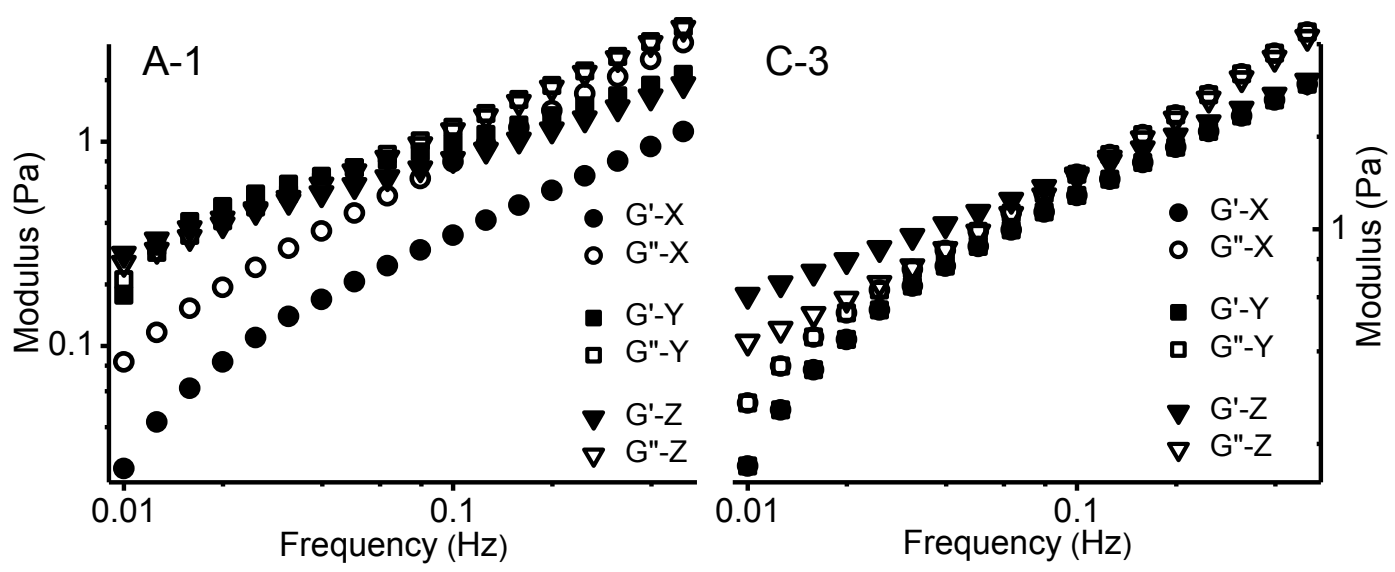

Fig. 7. Storage (G') and loss (G") moduli from frequency sweeps, respectively $X$ and $Y$, occurring before and after Step 1 flow; Z occurred after Step 2 decreasing flow (All applied after creep/recovery, Fig. 6). Note different ordinate scales in formulations A-1 (left) and C-3 (right).

Fig. 4-7 indicate that the PF/filler formulations exhibit complex behavior with the ability to form, or not form, viscoelastic fluids that exhibit some type of network structure. The formation of network structures correlated to the occurrence of the distinct viscosity recovery observed under decreasing shear (Step 2 flow). Fig. 4 demonstrates that this occurred for all corn cob formulations, regardless of particle size, but only for alder bark and walnut shell formulations containing the smallest particle size. The great challenge, not addressed here, is to determine the origin of this network behavior. Perhaps some of these observations could be explained by high shear disintegration of filler aggregates, remembering that all filler (and wheat flour) particles exceed 1 micron in size. So while soluble polymer in the liquid medium could interact with filler and flour particle surfaces, these particles are not expected to exhibit Brownian, or colloidal, interactions. Nevertheless, these various formulations clearly exhibit filler-specific effects where corn cob residue is distinct, and alder bark and walnut shell behave quite similarly, Fig. 4. Filler-specific effects could influence colloidal behavior in the liquid medium, discussed below.

As mentioned previously, PF chains in these formulations likely form entanglements and they are known to develop associations via hydrogen bonding among phenolic hydroxyls [9]. Phenolic associations among PF chains occur as a function of $\mathrm{NaOH}$ levels used in the highly alkaline resin. It is this high alkalinity that could create colloidal effects that are specific to the fillers used here. Aqueous alkali is known to promote the release of xylan and lignin fragments from within lignocellulose [15], not to mention gluten proteins and polysaccharides (amylose, amylopectin, and xylan) from wheat flour [10-12]. Under this scenario one could envision highly 
complex colloidal interactions mediated by soluble lignin, polysaccharides, and proteins, all among associating PF chains. Such colloidal phenomena would occur within the liquid medium that surrounds the massive, non-colloidal filler and wheat flour particles.

\subsection{Chemical composition of fillers}

Insight on potential colloidal effects is available from the chemical compositions of the respective fillers, Table 2 . Relative to alder bark and walnut shell, the distinct character of corn cob residue is seen in the xylan contents. Corn cob residue is produced from the acid catalyzed, steam digestion of corn cob where xylose is converted to the industrial monomer furfural. As a result of alkaline extraction (comparing Ex-free to Ex\&AE-free), it is seen that alder bark and walnut shell would likely release xylans into the PF medium, whereas corn cob residue would release very little. Likewise, the estimated alkaline leachates suggest that corn cob residue would release substantially more lignin (see acid insoluble lignin). Furthermore, corn cob residue contains much more solvent extractables (see soxhlet extractives). Due to the prior steam digestion of corn cob residue, these extractives are not expected to resemble the lipophilic compounds typical for materials like alder bark and walnut shell. For example, the glucan content in the estimated alkaline leachate suggests that corn cob residue might release significant quantities of glucose (or glucan) into the PF resin.

\section{Table 2}

Chemical constituents of alder bark (A), walnut shell (W), and corn cob residue (C).

\begin{tabular}{|c|c|c|c|c|c|c|c|c|c|}
\hline & & $\begin{array}{c}\text { Soxhlet } \\
\text { extractives }\end{array}$ & Glucan & Xylan & $\begin{array}{c}\text { Acid } \\
\text { insoluble } \\
\text { lignin }\end{array}$ & Arabinan & Galactan & Mannan & $\begin{array}{c}\text { Acid } \\
\text { soluble } \\
\text { lignin }\end{array}$ \\
\hline$A$ & & 9.4 & & & & & & & \\
\hline W & & 4.5 & & & & & & & \\
\hline C & & 25.5 & & & & & & & \\
\hline \multirow{3}{*}{ Ex-free } & $A$ & & 26.4 & 16.8 & 31.3 & 1.0 & 0.8 & 0.6 & 1.4 \\
\hline & W & & 26.9 & 22.0 & 31.6 & 0.5 & 1.2 & 0.1 & 2.1 \\
\hline & C & & 46.7 & 0.9 & 27.2 & 0.1 & 0.1 & 0.1 & 0.4 \\
\hline \multirow{3}{*}{$\begin{array}{c}\text { Ex\& } \\
\text { AE-free }\end{array}$} & $A$ & & 26.0 & 10.8 & 26.2 & 0.8 & 0.6 & 0.5 & 0.9 \\
\hline & W & & 25.4 & 13.1 & 25.6 & 0.3 & 0.9 & 0.1 & 1.6 \\
\hline & C & & 40.6 & 0.4 & 17.7 & 0.1 & 0.0 & 0.1 & 0.2 \\
\hline \multirow{3}{*}{$\begin{array}{l}\text { Alkaline } \\
\text { leachate }\end{array}$} & $A$ & & 0.4 & 6.0 & 5.1 & 0.2 & 0.2 & 0.1 & 0.5 \\
\hline & W & & 1.5 & 8.9 & 6.0 & 0.1 & 0.3 & 0.0 & 0.5 \\
\hline & C & & 6.0 & 0.5 & 9.5 & 0.0 & 0.1 & 0.0 & 0.2 \\
\hline
\end{tabular}

\section{Discussion}


Considering that the fillers and wheat flour will release polymeric and monomeric compounds under alkaline conditions, the formation of colloidal particles within the PF medium seems quite plausible. Complex rheological behavior within these adhesive formulations could arise from colloidal effects within the PF medium and also from polymeric adsorption onto filler particle surfaces. In the latter case, it is known that PF chains adsorb onto cellulosic/lignocellulosic surfaces as a strong function of $\mathrm{pH}$ and ionic strength [16, 17]. Furthermore, complexes between PF and non-ionic water soluble polymers are known to hinder PF adsorption [17].

The rheological complexity of these PF/filler formulations is apparent and this work demonstrated that shearing stimulates the formation of viscoelastic network structure within the fluids. Not determined here are the minimum shear rates required to stimulate the transformations, and how these relate to the shear rates employed during industrial manufacture. Also unknown is the stability of these structures and the time periods over which they may evolve. Finally, perhaps the most important question is if and how these structures impact adhesive performance, with possible effects ranging from adhesive tack, to bondline moisture retention, and ultimately into the morphology and toughness of the cured bondlines.

\section{Conclusions}

PF/wheat flour/organic filler formulations are well established technologies for the commercial manufacture of veneer-based wood composites, and yet much remains unknown about structure within these fluids and the corresponding impact on adhesive performance. It was shown that viscoelastic network structures form within the liquid formulations as a function of shear history, organic filler type, and filler particle size. The precise nature and origin of these effects is unknown but could involve disintegration of filler particle aggregates on a non-colloidal scale, and/or colloidal effects within the liquid PF medium. In the latter case colloidal structures could form from the proteins, polysaccharides, and lignins that emanate from wheat flour and filler particles under the alkaline conditions within the PF resin. These natural polymers will likely interact with individual PF chains, and among clusters of PF chains associating by phenolic secondary interactions. Many implications for impact on industrial practice are feasible and should be the subject of future research.

\section{Acknowledgments}


This project was supported by the Wood-Based Composites Center, a National Science Foundation Industry/University Cooperative Research Center (Award \# 1034975).

\section{References}

[1] Sellers T. Plywood and adhesive technology. New York: M. Dekker; 1985.

[2] D907. Standard terminology of adhesives. West Conshohocken, PA: ASTM International; $2012 a$.

[3] Robertson J.E., Robertson R. Review of filler and extender quality evaluation, Forest Prod J 1977;27:30-8.

[4] Goldstein I.S., editor. A plywood review and its chemical implications. Proceedings of the Wood Technology: Chemical Aspects; 1977; Washington, D.C. Washington, D.C.: American Chemical Society; 1977.

[5] Ebewele R.O., River B.H., Koutsky J.A. Relationship between phenolic adhesive chemistry and adhesive joint performance: effect of filler type on fraction energy, J Appl Polym Sci

1986;31:2275-302.

[6] D1105. Standard test method for preparation of extractive-free wood. West Conshohocken, PA: ASTM International; 1996.

[7] Sluiter A., Hames B., Ruiz R., Scarlata C., Sluiter J., Templeton D., et al. Determination of structural carbohydrates and lignin in biomass. Golden, CO: National Renewable Energy Laboratory; 2008.

[8] Ehrman T. Determination of acid-soluble lignin in biomass. Golden, CO: National Renewable Energy Laboratory; 1996.

[9] Haupt R.A., Sellers T.J. Characterizations of phenol-formaldehyde resol resins, Ind Eng Chem Res 1994;33:693-7.

[10] Goesaert H., Brijs K., Veraverbeke W., Courtin C., Gebruers K., Delcour J. Wheat flour constituents: how they impact bread quality, and how to impact their functionality, Trends Food Sci Tech 2005;16:12-30.

[11] Van Der Borght A., Goesaert H., Veraverbeke W.S., Delcour J.A. Fractionation of wheat and wheat flour into starch and gluten: overview of the main processes and the factors involved, J Cereal Sci 2005;41:221-37.

[12] Alexander R.J., Krueger R.K., inventors; Krause Milling Company, assignee. Plywood adhesives using amylaceous extenders comprising finely ground cereal-derived high fiber byproduct. United States patent US 4070314. 1978.

[13] Potanin A. Thixotropy and rheopexy of aggregated dispersions with wetting polymer, J Rheol 2004;48:1279-93.

[14] Mewis J., Wagner N.J. Thixotropy, Adv Colloid Interfac 2009;147:214-27.

[15] Glasser W.G., Kaar W.E., Jain R.K., Sealey J.E. Isolation options for non-cellulosic heteropolysaccharides (HetPS), Cellulose 2000;7:299-317.

[16] Johansson K., Larsson A., Ström G., Stenius P. Adsorption of phenol-formaldehyde resin onto cellulose, Colloids Surface 1987;25:341-56.

[17] Stack K., Dunn L., Roberts N. Adsorption studies of phenolformaldehyde resin onto cellulose fibres, Colloids and Surface A 1993;70:23-31. 